\title{
Processes and structures in intergenerational programs: a comparison across different types of programs
}

\author{
Jiska Cohen-Mansfield ${ }^{1,2,3}$ (1) and Aline Muff ${ }^{2}$ \\ ${ }^{1}$ Department of Health Promotion, School of Public Health, Sackler Faculty of Medicine, Tel Aviv University, Tel Aviv, Israel \\ ${ }^{2}$ Minerva Center for Interdisciplinary Study of End of Life, Tel Aviv University, Tel Aviv, Israel \\ ${ }^{3}$ The Herczeg Institute on Aging, Tel Aviv University, Tel Aviv, Israel
}

Abstract

Objectives: Given the widely acknowledged benefits of intergenerational programs (IGPs), we compared processes and structures across different IGP types and explored potential areas for improvement.

Design: Thirteen IGPs were classified into three types: arts, learning, and assistance programs. Data were collected through direct structured interviews and analyzed quantitatively and qualitatively.

Setting: Participants were recruited from community-based IGPs in the greater Tel Aviv area of Israel.

Participants: Eighty-four older participants (OPs), 97 younger participants (YPs), and 21 organizers were interviewed.

Measurements: Questions included participant demographics as well as closed- and open-ended questions regarding processes based on the Impact of Intergenerational Programs Questionnaire (IIPQ).

Analysis: Responses to closed-ended questions were compared among IGP types and age groups using two-way ANOVAs for ordinal data, and chi-squares for nominal data. Responses to open-ended questions by OP, YP, and program organizers about potential areas for improvement were analyzed using thematic analysis.

Results: Processes in need of improvement were preparation and guidance, length of program participation, and monitoring of activities, which differed across IGP types and age groups. These processes were related to broader structural problems such as lack of resources, organizers' poor employment conditions, and inadequate public services for older persons in Israel.

Conclusions: Our study highlights the complex relationships between IGP types, processes, and structures. IGP processes and goals can be hindered by structural variables such as insufficient funding, infrastructure, and public services for older adults.

Key words: intergenerational programs, younger adults, older adults, public services for older adults, program evaluation

\section{Introduction}

The detrimental physical and mental health impact of social isolation and loneliness on older adults has been widely reported in the literature (CohenMansfield et al., 2016; Santini et al., 2020; Sundström et al., 2020), while these are increasingly identified as issues with which younger people struggle as well (Child and Lawton, 2019; Jeste et al., 2020; Matthews et al., 2016; Pitman et al., 2018). Intergenerational programs (IGPs) can address

Correspondence should be addressed to: Jiska Cohen-Mansfield, Department of Health Promotion, Tel Aviv University, P.O.B. 39040, Ramat Aviv, Tel Aviv, 6139001, Israel. Tel: 972-3-6407337, Fax: 972-3-6407339. Email: jiska@post tau.ac.il. Received 10 Jan 2021; revision requested 03 Feb 2021; revised version received 06 Jun 2021; accepted 14 Jun 2021. First published online 09 August 2021 social isolation and loneliness for young and old alike (e.g. Parkinson and Turner, 2019).

IGPs are designed to: (1) foster positive relationships and mutual understanding between generations (Canning et al., 2018); (2) reduce negative perceptions of the other generation (Gaggioli et al., 2014; Meshel and McGlynn, 2004); and/or (3) improve physical and mental health outcomes among participants (Murayama et al., 2015; Perry and Weatherby, 2011), decrease loneliness (Aguilera-Hermida et al., 2020), and increase social connection (MacCallum et al., 2010).

Research about IGPs has focused mostly on the impact of interventions on younger persons (YP) and older persons (OP) (Gualano et al., 2018; Voglino et al., 2017). While most studies report 
clear benefits for YP and OP who participate in IGPs (Holmes, 2009; Jarrott and Bruno, 2007; Yasunaga et al., 2016), benefits may be hindered by infrequent contact (Martins et al., 2019) and divergent needs of participants (e.g. different energy and activity levels between and within generations) (Stremmel et al., 1994).

IGP "processes" are defined as sequences of actions (e.g. activity format, preparation and training, evaluation). Processes are the means through which programs are implemented, and the evaluation of different process variables, such as the availability of guidance and activity location, can elucidate their influence on programs and outcomes (Steckler and Linnan, 2002). Process variables characterize the nature of sequences of actions within an intervention. We define process variables in this article as characteristics of the IGP intervention process.

Processes that were previously reported to improve intergenerational activities include consistent scheduling and explicit encouragement of intergenerational interaction (Epstein and Boisvert, 2006; Jarrott et al., 2019; Lou and Dai, 2017). Another process that is frequently noted is ongoing training of IGP organizers (DeVore et al., 2016; Gigliotti et al., 2005), and preparatory and continuing guidance for participants (Cohen-Mansfield and Jensen, 2017; Jarrott et al., 2019; McAlister et al., 2019). In addition, ongoing program evaluation has been mentioned as an important process (DeVore et al., 2016; Epstein and Boisvert, 2006).

Previous research demonstrated that different IGP activities such as arts, learning, or providing assistance can yield different benefits (Bullock and Osborne, 1999; Lokon et al., 2012; Newman and Hatton-Yeo, 2008). Similarly, in a separate paper, we found significant differences among different types of IGPs as to the characteristics of participants they attract, reported benefits, and challenges (Cohen-Mansfield and Muff, 2021).

While our findings affirmed the important contribution of IGPs in enhancing OP and YP lives, there remains a continuing need to study process variables within IGPs since there is a dearth of literature about them. To the best of our knowledge, differences in processes across different IGP types have not been researched previously. Such research is needed to clarify how processes that underlie different types of IGPs may hinder or promote their goals, and how IGPs can be improved. In this article, we examine whether different IGPs which vary in goals and content, also differ in their process variables.
The specific research questions are:

1. How do process variables differ across different types of community-based IGPs and between the reports of OP and YP? (quantitative analysis)

2 . Which processes are perceived as needing improvement from the perspective of participants and organizers? (qualitative analysis)

\section{Methods}

\section{Participants and recruitment}

We contacted nationwide and local municipal offices which offer IGPs, as well as a variety of not-forprofit organizations, to identify active IGPs. The inclusion criterion for IGPs was that they were directed and organized within the community in contrast to being school-based, which we studied previously (Cohen-Mansfield and Jensen, 2017). The inclusion criterion for OP and YP was current participation in a community-based IGP at the time of the interview. In addition, we recruited organizers who ran IGPs we wished to study.

We distinguished between three communitybased group types based on their content and goals:

Arts group: This group included an intergenerational community theater, and an intergenerational dance program.

Learning group: This group included an intergenerational Bridge game, a photography course, and Jewish text study sessions.

Assistance group: This group focused on assisting OP one-on-one, and included a program in which YP visited $\mathrm{OP}$ at their homes, and spent time together in various activities; a program in which YP documented an OP's life story; two programs in which YP visited older Holocaust survivors; a program in which Israel Defense Force soldiers visited OP in their homes or at a senior club; a program in which YP taught OP how to use a computer; a program that sought to foster long-term personal intergenerational relationships; and a program in which YP assisted OP in taking care of their pets. While it was anticipated that YP and OP would engage in social interaction as part of the assistance programs, tasks such as cleaning, and other housekeeping chores, driving OPs to doctor appointments, and the like, were not within the purview of these programs.

While all IGPs under study aimed to generally improve intergenerational relationships, arts programs focused on creating cooperation between the two generations, learning programs focused on learning together and on developing specific skills, and assistance programs were geared toward 
lending certain types of support to OP in their environment and alleviating loneliness through regular visits. Altogether, the study involved 84 OP, 97 $\mathrm{YP}$, and 21 organizers.

\section{Procedures}

The study was approved by the ethics committee of Tel Aviv University. After participants gave informed written consent, interviews were either conducted by trained interviewers $(n=167)$ or self-administered $(n=35)$. Self-administered questionnaires were completed online or in writing using the same questions. Research staff contacted interviewees who did not answer all of the questions or to eliminate missing data.

\section{Assessments}

The structured interviews included closed- and open-ended questions. All interviews began with demographic and background questions including age, gender, and education. Information concerning the subjective health of OP was based on participants' evaluation of their current health, ranging from 1 (poor) to 5 (excellent), based on the global self-rated-health item on the SF-36 (Ware and Sherbourne, 1992).

The Impact of Intergenerational Programs Questionnaire (IIPQ), based on Cohen-Mansfield and Jensen (2017), included the following closed-ended questions concerning the process: How long have you been participating in the activity with the other (age) group? How often do you meet with the other (age) group? What is the duration of a typical meeting? Did you receive any guidance/training before the activity began? Do you receive guidance throughout the duration of the activity? Would you be interested in changing the activity location? Do you worry about issues in your relationship with member(s) of the other generation? Responses were elicited through ordinal scales or binary (Yes/No) response options.

Process issues were explored through the following open-ended questions: What are the difficulties or problems that arise for you within the activity with the other (age) group? What would you prefer to do with the other (age) group? In your opinion, how can the activity be improved? Organizers were asked about issues that came up when coordinating and organizing the activity. The questions are summarized in Table S1 (published as Supplementary material online attached to the electronic version of this paper).

\section{Statistical approach}

In order to answer the research question, "How do process variables differ across different types of
IGPs, and between reports by OP and YP?", we compared the three IGP types using two-way ANOVA for ordinal data, and chi-square for nominal data.

\section{Qualitative approach}

Responses to open-ended questions were transcribed by interviewers during the interviews. Interviewers also noted interviewees' reflective comments, documenting observations and impressions gained therefrom. The qualitative analysis focused on the question, "Which processes are perceived as needing improvement from the perspective of participants and organizers?" We used thematic analysis to organize our data from the interviews into themes and patterns (Braun and Clarke, 2006). Themes were defined according to whether they captured an idea relevant to our research questions. To avoid single-researcher bias, different members of the staff conducted their analysis independently (Denzin, 1989). After several rounds of coding, recoding, and comparative analysis, the research team decided on a list of themes and sub-themes on the basis of similarity and overlap.

\section{Results}

\section{Quantitative analysis}

DEMOGRAPHICS

Of OP, $82 \%$ were female, compared to $67 \%$ among YP. The average age of OP was 77.2, whereas YP had an average age of 23.9. The majority of participants were unmarried, $68 \%$ of OP and $94 \%$ of YP. The average number of years of education was 13.1 for OP and 12.3 for YP. Participant demographic background is presented in Table 1 .

\section{Process VARIABles}

Two-way ANOVA with the factors of "program type" and "participant age group" was used to compare the process variables that were measured by ordinal scales (Table 2). For the length of participation in the program, there was a significant interaction between group type and participant group $(\mathrm{p}=.01)$, where the longest participation over a year - was reported by OP in the assistance program, whereas the shortest - around 3 months was reported by OP in the learning program. This interaction is also reflected in the finding that in the assistance group, OP had a significantly longer period of participation compared to YP $(\mathrm{p}<.001)$, while this difference was not significant in the arts or learning groups $(\mathrm{p}=.47, \mathrm{p}=.66$ respectively). The group type effect was also statistically significant $(\mathrm{p}<.001)$, with post hoc comparison indicating that 
Table 1. Demographic background variables (YP, OP, and organizers) percentages or mean (SD)

\begin{tabular}{|c|c|c|c|c|c|c|c|}
\hline & Participant & ARTs & LEARNING & Assistance & Total & $F / X^{2}$ & $P$ \\
\hline \multirow[t]{3}{*}{$N$} & Older & 32 & 15 & 37 & 84 & & \\
\hline & Younger & 17 & 16 & 64 & 97 & & \\
\hline & Organizer & 1 & 5 & 15 & 21 & & \\
\hline \multirow[t]{3}{*}{ Sex (\%Female) } & Older & $81 \%$ & $80 \%$ & $84 \%$ & $82 \%$ & $\chi_{(2)}^{2}=0.13$ & .936 \\
\hline & Younger & $94 \%$ & $38 \%$ & $67 \%$ & $67 \%$ & $\chi_{(2)}^{2}=11.96$ & .003 \\
\hline & Organizer & $100 \%$ & $60 \%$ & $67 \%$ & $67 \%$ & & \\
\hline \multirow[t]{3}{*}{ Age } & Older & $76.5(5.4)$ & $75.3(8.9)$ & $78.7(7.7)$ & $77.2(7.2)$ & $F_{(2,79)}=1.50$ & .231 \\
\hline & Younger & $25.9(12.0)$ & $21.3(9.3)$ & $24.0(5.3)$ & $23.9(7.6)$ & $F_{(2,93)}=1.55$ & .218 \\
\hline & Organizer & $67.0(-)$ & $46.4(18.1)$ & $39.9(15.2)$ & $42.9(16.3)$ & & \\
\hline \multirow[t]{3}{*}{ Years of education } & Older & $14.7(6.5)$ & $14.3(7.5)$ & $11.3(4.0)$ & $13.1(5.8)$ & $F_{(2,66)}=3.06$ & .054 \\
\hline & Younger & $12.5(5.5)$ & $10.1(4.2)$ & $12.8(4.0)$ & $12.3(4.4)$ & $F_{(2,92)}=2.50$ & .088 \\
\hline & Organizer & $15.0(-)$ & $17.0(3.8)$ & $14.9(6.9)$ & $15.4(6.1)$ & & \\
\hline
\end{tabular}

length of participation in the learning group was significantly shorter than in the arts $(\mathrm{p}<.01)$ and assistance groups $(\mathrm{p}<.001)$. The differences between the three IGP types accounted for $19.3 \%$ of the variance in length of participation (i.e. $\eta_{\mathrm{p}}{ }^{2}=0.193$ ), while the interaction effect accounted for $5.3 \%$.

Reported meeting duration of program sessions significantly differed by IGP type $(p=.02)$. It was longest for the arts programs (over $90 \mathrm{~min}$ on average) and shortest for the assistance programs (less than $90 \mathrm{~min}$ on average). The difference between reports of younger versus older participants was not significant. No significant differences were found concerning meeting frequency, which averaged about once a week.

Significant differences were found both as to activity type, and age group in regard to receiving guidance during the course of the activities. Those in the arts programs reported receiving guidance several times a month compared to those in the assistance group reporting receiving guidance less than once a month $\left(\mathrm{p}<001\right.$ for group type, $\left.\eta_{\mathrm{p}}^{2}=0.123\right)$. OP received significantly less guidance than YP $\left(p<.001, \eta_{p}^{2}=0.098\right)$, and, although the interaction term did not reach statistical significance $(\mathrm{p}=.07)$, OP in the assistance group tended to receive almost no guidance at all, whereas $\mathrm{YP}$ in the arts group received the most - almost once a week.

Nominal variables were analyzed using chisquare to compare responses across activity group types and across age groups (Table 3). Regarding guidance received prior to program commencement, differences among IGP types were not statistically significant, but YP (62.9\%) were significantly more likely to receive such guidance than OP $(28.0 \%, \mathrm{p}<.001, \phi=0.348$, indicating a medium effect size based on Kim (2017).

Satisfaction with the location did not differ with IGP type, but OP were significantly more likely to be satisfied (95.1\%) than YP $(83.5 \%, \mathrm{p}<.05$, $\phi=0.184$, small effect).

Worry about difficulties in the intergenerational relationship was significantly $(\mathrm{p}=.01)$ more likely to occur in the assistance programs (32.3\%) than in the other programs and more likely to occur among YP $(37.5 \%)$ than among OP $(8.6 \%, \mathrm{p}<.001$, $\mathrm{V}=0.228, \phi=0.335$ respectively, both medium effects).

\section{Qualitative analysis}

The goal of the qualitative analysis was to deepen our understanding of the quantitative findings, and to address the research question, "Which processes are perceived as needing improvement from the perspective of participants and organizers?" We organized the themes identified in the data according to processes and structures, the latter having been articulated by participants.

\section{Processes}

YPs' lack of consistent attendance This was manifested by YP showing up late, missing meetings, or not notifying others of late arrival in a timely manner. Explanations involved YPs' other commitments such as studies, work, or family: "I cannot come to them more [than I already do], I also work and do other things" (\#132, age 20-female (F), assistance), or, "[I]t saddens OP that the students come less. .... [T] hey come less often because of the exams, because of studies" (\#113, 50-F-organizer (O), learning).

Need for monitoring Inconsistent attendance and tardiness may have been related, at least in part, to a general lack of monitoring, as one OP suggested, “... they [organizers] never checked them [YP] ... They [organizers] think that [one YP] keeps coming 
Table 2. Process variables - a comparison across programs and age groups (ordinal variables)

\begin{tabular}{|c|c|c|c|c|c|c|c|c|}
\hline & & & & & & $\mathrm{F}$ & $\mathrm{P}$ & $\mathbf{H}_{\mathbf{p}}{ }^{2}$ \\
\hline & & & & & & \multicolumn{3}{|c|}{ Factor A (between programs) } \\
\hline & & $(\mathrm{n}=49)$ & $(\mathrm{n}=31)$ & $(\mathrm{n}=101)$ & $(\mathrm{n}=181)$ & \multicolumn{3}{|c|}{ Factor B (between age groups) } \\
\hline & & $M(S D)$ & $M(S D)$ & $M(S D)$ & $M(S D)$ & \multicolumn{3}{|c|}{$\mathrm{A} \times \mathrm{B}$} \\
\hline \multirow[t]{3}{*}{ Length of participation ${ }^{1}$} & Older & $3.1(1.0)$ & $1.9(1.4)$ & $4.4(1.6)$ & $3.5(1.7)$ & $F(2,169)=20.26$ & $<.001$ & 0.193 \\
\hline & Younger & $2.9(0.6)$ & $2.1(1.1)$ & $3.1(1.4)$ & $2.9(1.3)$ & $F(1,169)=3.42$ & .066 & 0.020 \\
\hline & Total & $3.1(0.9)^{\text {a } * *}$ & $2.0(1.2)$ & $3.6(1.6)^{\text {a } * * *}$ & $3.2(1.5)$ & $F(2,169)=4.72$ & .010 & 0.053 \\
\hline \multirow[t]{3}{*}{ Meeting duration ${ }^{2}$} & Older & $3.1(0.2)$ & $2.9(0.7)$ & $2.7(0.7)$ & $2.9(0.6)$ & $F(2,173)=3.95$ & .021 & 0.044 \\
\hline & Younger & $3.2(0.4)$ & $3.1(0.9)$ & $3.0(0.7)$ & $3.0(0.7)$ & $F(1,173)=3.75$ & .054 & 0.021 \\
\hline & Total & $3.1(0.3)$ & $3.0(0.8)$ & $2.9(0.7)$ & $3.0(0.7)$ & $F(2,173)=0.15$ & .867 & \\
\hline \multirow[t]{3}{*}{ Meeting frequency ${ }^{3}$} & Older & $5.7(0.5)$ & $5.7(0.5)$ & $5.8(0.7)$ & $5.7(0.6)$ & $F(2,171)=0.58$ & .559 & \\
\hline & Younger & $5.8(0.4)$ & $5.6(1.0)$ & $5.9(1.0)$ & $5.8(0.9)$ & $F(1,171)=0.07$ & .797 & \\
\hline & Total & $5.7(0.4)$ & $5.6(0.8)$ & $5.8(0.9)$ & $5.8(0.8)$ & $F(2,171)=0.29$ & .745 & \\
\hline \multirow[t]{3}{*}{ Guidance during the activity ${ }^{4}$} & Older & $2.1(1.4)$ & $2.5(1.6)$ & $1.2(0.4)$ & $1.7(1.2)$ & $F(2,158)=11.09$ & $<.001$ & 0.123 \\
\hline & Younger & $3.4(1.2)$ & $2.6(1.5)$ & $2.4(1.2)$ & $2.7(1.3)$ & $F(1,158)=17.14$ & $<.001$ & 0.098 \\
\hline & Total & $2.6(1.5)^{\mathrm{b} * *}$ & $2.6(1.5)$ & $1.9(1.2)$ & $2.2(1.3)$ & $F(2,158)=2.69$ & .071 & 0.033 \\
\hline
\end{tabular}

${ }^{*} \mathrm{p}<.05,{ }^{* *} \mathrm{p}<.01,{ }^{* * *} \mathrm{p}<.001$.

a Post hoc, Scheffe, significant difference compared to "learning" group.

b Post hoc, Scheffe, significant difference compared to "assistance" group.

${ }^{1} 1$ - Less than 3 months, 2 - 3-6 months, $3-6$ months -1 year, 4 - a year, 5 - one and a half- 2 years, 6 - over 2 years.

21 - up to half an hour, 2 - up to 1 hour, $3-1$ hour and a half-2 hours, 4 - more than 2 hours.

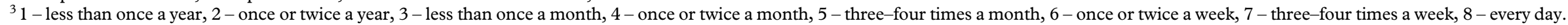

${ }^{4} 1$ - not at all, 2 - less than once a month, 3 - less than once a week, 4 - once or twice a week.

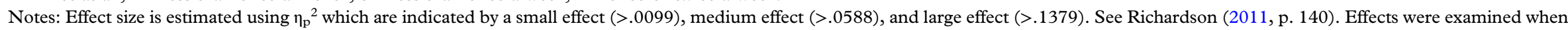
$\mathrm{p} \leq .07$; medium and larger effects are bolded. 
Table 3. Process variables - a comparison across programs and age groups (nominal variables)

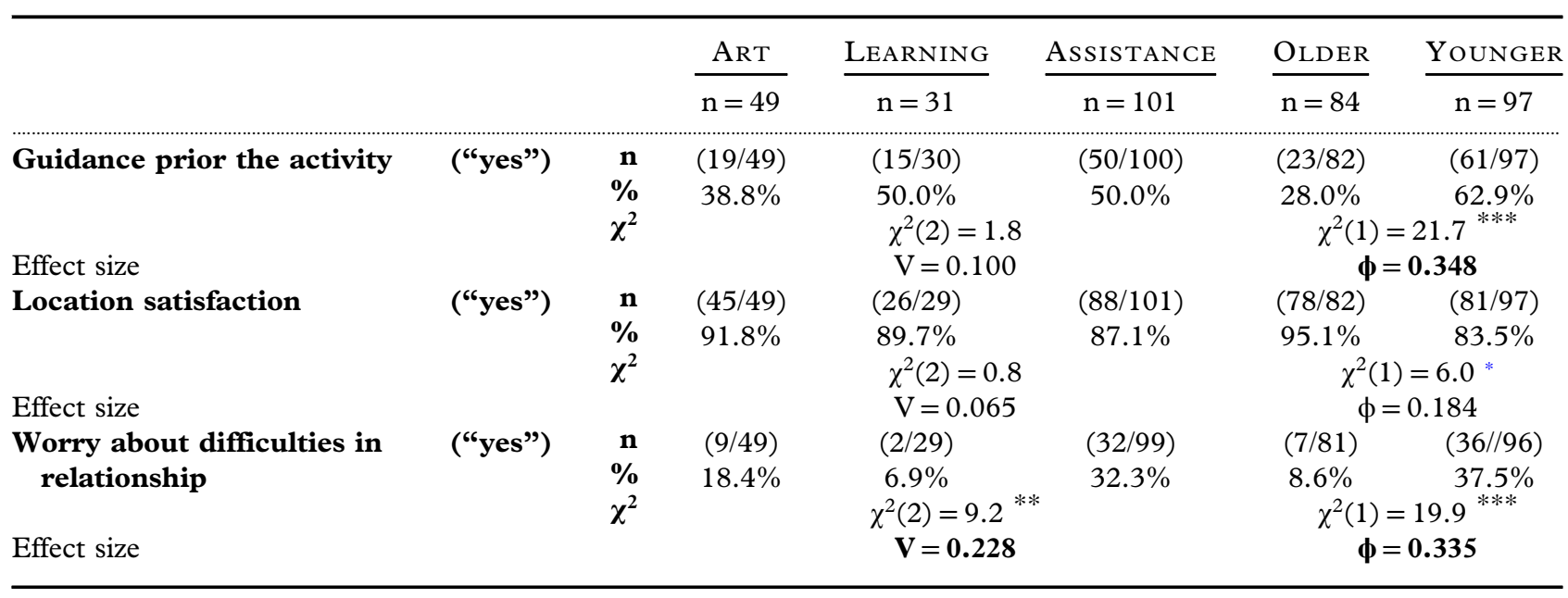

${ }^{*} \mathrm{p} \leq .05^{* * \mathrm{p}} \leq .01^{* * *} \mathrm{p} \leq .001$.

According to Kim (2017, p. 254), for chi-squared tests with $1 \mathrm{df}$, the criteria for small, medium, and large effects are $0.10,0.30$, and 0.50 , respectively; for $2 \mathrm{df}$, the respective numbers are: $0.07,0.21$, and 0.35 .

[even though he does not]. There is a lack of tracking of the activity" (\#24, 90-M, assistance).

Stereotyping and lack of intergenerational understanding as to others' needs and expectations Some OP expected IGPs to meet their needs in ways that went beyond the scope of the IGP. This was particularly common in assistance programs that aimed to support OPs through regular visits, but did not include housekeeping and transportation services. For example, "They $[\mathrm{OP}]$ want the volunteers to do things they are not supposed to do, such as cleaning the house and doing errands. It is difficult for them to understand that the activity is social rather than ... concrete help" (\#71, 27-M-O, assistance), or, "It is difficult to set boundaries for them ... they want me to stay beyond the meeting time or to come to them even outside the hours of the activity" (\#28, 20-F-assistance).

One YP explained that OP did not seem to understand the purpose of the IGP and that the expectations were not properly established:

At first, she did not understand what I was doing there, [which contributed to] a distance between us, the expectations were not coordinated... Once I even washed the floor ... because she really did not understand what the purpose was... She knew people only when they came to her home to fix [things]... (\#86, 19-M, assistance)

The combination of YPs' insufficient attendance and OPs' uninformed expectations resulted in some OP and YP complaining about each other. While OP complained about YPs' lack of reliability and punctuality, YP criticized OP's mistaken expectations. One organizer offered an explanation of the dissonance:
It's hard for them [OP] to have a different way of doing things; the concept of time is different... OP expect punctuality and from the point of view of the [young] volunteer, there is greater flexibility, and that creates ...

a real inter-generational gap. (\# 1, 31-F-O, assistance) Intergenerational dissonance may have been amplified by participants' stereotypes. One YP described $\mathrm{OP}$ as emotionally unstable and inconsistent: "They [OP] are unpredictable. There are a lot of cases where they can seem terribly apathetic and not caring about anything and suddenly, they become very sensitive about the smallest matter." (\#91, 18$\mathrm{F}$, assistance). Further, some OP described YP as unreliable: "The adults come on time and the young people, you know how it is, everyone comes whenever he wants and it is very disruptive" (\#96, 69$\mathrm{F}$, arts).

Lack of preparation and guidance Lack of sufficient skills and experience among YP, both prior to and during participation was reported in all IGP types. YP experienced difficulties "coping with emotional states such as depression [among] OP... understanding [OP's] physical and cognitive deterioration that can affect the relationship" (\#23, 37-F-O, assistance). Thus, insufficient guidance was repeatedly mentioned: " $[\mathrm{T}]$ he volunteers [should] receive [at least] minimal guidance, how to behave, what they should not do..." (\#24, 90-M, assistance). OP were also unprepared for and unfamiliar with the various programs' scope and expectations for their behavior, as one organizer explained: "As for the [older] adults - they are probably the least prepared. They often show concerns. ....[T] hey do not always remember all the conditions and do not exactly understand" (\#170, 32-F-O, assistance). 
Consequently, some OP expected YP to assist them in tasks that went beyond the scope of the program: "[I would like to] extend [the meeting], at least for 4 hours together. I would like for example that she [will] read articles to me, help me with filing, office work that I cannot do alone" (\#65, 87-M, assistance).

Participation in program too short or sporadic Some organizers and participants stated that the participation designated to achieve IGPs' stated objectives was too short, for example, "We had projects ... [in which] volunteers serving a year of service did not succeed in writing down [the OP's life story] and that created a situation where there was no product" (\#4, 36-M-O, assistance), or,

Someone volunteers to help us ... and that's [worth] nothing, she shows you one thing and leaves. ... [Y] ou can ask them one question, and the session is finished.

$\ldots$.. [I]t is short and sporadic. (\#122, 81-F, assistance) Many OP expressed the wish to continue or to expand the activity: "It's a very limited time together, I'd like to have more" (\#88, 74-F, assistance).

\section{STRUCTURES}

Lack of adequate facilities One organizer complained that some of the facilities where IGPs took place were inadequate, e.g. insufficient space to accommodate activities for larger groups, and problematic accessibility for OP (\#108, 61-F-O, assistance). Another organizer noted the absence of a room in which to conduct orientations for volunteers as to age-related differences and vulnerabilities, and interpersonal skills (\#21, 63-F-O, assistance).

Lack of transportation In some instances, inadequate public transportation and commuting distance were logistical problems that affected YPs' willingness to visit OP, "Sometimes the girls do not agree to take two buses, sometimes they have no choice" (\#19, $62-\mathrm{F}-\mathrm{O}$, assistance). Travel was a barrier to extending programming to areas of Israel where public transportation is less frequent than in urban centers. Organizing transport for the OP with limited mobility was reported to be difficult, irrespective of region (\#95, 41-F-O, assistance).

Budgetary and staff shortfalls Many problems were attributed to insufficient funding. For example, one organizer explained that there was no budget for training and guidance: " $[\mathrm{Need}$ for] [m]ore training, more investment - had there been more investment in the field, in volunteers and OP, in mentoring and guidance, [our situation] would have been much better" (\# 1, 31-F-O, assistance).
As a consequence, there was a staff shortage: "We need more coordinators, more marketing people, more manpower in short ... and for that we need more resources" (\# 2, 26-M-O, assistance).

Problematic employment conditions In addition to, or as a consequence of budgetary difficulties and understaffing, organizers complained about difficult working conditions: "The salary ... is relatively low, and not in line with the [wage] agreement for social workers" (\#14, 29-F-O, assistance), or, "The coordinators are unsalaried volunteers, which makes it very difficult. We are a national organization with one and a half salaried workers, no more. We reach 450 OP a year, but there are 20,000 lonely OP in the country" (\#71, 27-M-O, assistance). Low salaries may explain the high turnover among organizers, "The coordinators change often ... maybe because of the salary" (\#14, 29-F-O, assistance). Similarly, organizers worry about job security: "Last year, I began with a certain number of young women, and fewer completed the assignment, so my job was cut during the year. There is no job security" (\#21, 63F-O, assistance).

Understaffing caused the available personnel to be overworked: "I run around from one place to another... using public transportation most of the time... it can be exhausting ... inefficient and very time consuming... I handle phone calls non-stop because OP call me constantly..." (\#14, 29-F-O, assistance).

Insufficient public services for OP IGPs take place in the context of insufficient and inadequate social services. Therefore, YP may find themselves interacting with OP who have important unmet needs, the nature of which can be beyond the scope of the particular IGP program's mission, and may be beyond the skill set or comfort zone of the average YP. For example, some YP reported that they were asked to help with daily errands (shopping, doctor visits, phone calls, etc.): "It is very frustrating for her to cope with public institutions, the internet, television and telephone, and she often needs external help, that is - from me" (\#145, 39-F, assistance), or, "To drive her $[\mathrm{OP}]$, to escort her to places, .... but (a) I'm not allowed to do this, and (b) It's not something that can be kept up [over time] ..." (\#140, 30-F, assistance).

In some instances, a YP may be the agent for uncovering what neither family members nor social welfare professionals have yet discovered:

Problems and distress are detected through YP because there are situations of disconnectedness in which there is almost no involvement of community representatives... This can involve discovery of violence towards OP, the detection of dementia. Reporting this sometimes results 
in community involvement... (\#21, 63-F-O, assistance)

The qualitative results indicate that processes and structures that underlie IGPs are interrelated and may influence their effectiveness and meaningfulness.

\section{Discussion}

The first research question inquired into differences in process variables among different IGPs, and between OP and YP. Some significant differences were found, suggesting that program types and participant characteristics have an important influence on process variables. A large effect size was found for the differences across programs in the length of participation, where participants reported having participated for the longest time period in assistance programs, and the shortest in learning programs. Since assistance programs were focused on developing a close relationship between one OP and one YP, a longer period of contact fit the program goal. Yet, there was a general perception in the qualitative findings, especially among OP, that the length of participation was insufficient. This may have been influenced by the expectations of OP who enrolled in the assistance programs with the goal of receiving ongoing assistance.

Reports concerning the level of guidance provided to participants differed between OP and YP, and across IGPs - both suggesting medium-size effects. Participants in arts and learning programs reported receiving the most guidance during activities, while those in assistance programs reported receiving the least. YP received significantly more guidance than OP. Similarly, significant differences were found in reporting receipt of guidance for participation prior to program commencement, with YP most likely to report receiving prior guidance, and OP least likely to report it. Perhaps organizers perceived OP as service recipients who did not require training, but perceived $\mathrm{YP}$ as service providers who required training, which is a common process in IGPs (Leedahl et al., 2019; Zucchero, 2010). The qualitative results demonstrated, however, that OP also needed guidance, at least as to orientation and expectations.

Reports of worry concerning potential difficulties in relationships between generations were relatively infrequent among OP (8.6\%) compared to over a third of YP (37.5). Such worry was most common in the assistance programs (32.3\%), with the lowest rates found in the learning programs $(6.9 \%)$. Age of participant and type of IGP each manifested a medium-size effect on such worries. The low level of relationship worry in the learning programs may have been a function of the parties focusing more on the learned content than on each other.

The second research question sought to detect processes perceived as needing improvement. Such processes were reported as a lack of consistent attendance and punctuality on part of some YP, while YP and organizers reported that some OP held expectations that went beyond the IGP's scope, such as the assumption that YP would perform housekeeping chores. These dynamics suggest that better preparatory guidance for YP and OP could improve program outcomes. In addition, there is an obvious need for monitoring IGP activity, including YP attendance, whether IGP activities are being performed, and the extent to which program goals are being met. The suggestion of monitoring is not meant to suggest failure, but intended to boost quality assurance and maximize IGP benefits. It is clear that IGPs yield substantial benefits for participants, as we have shown elsewhere (Cohen-Mansfield and Muff, 2021) and that program evaluation serves a supportive purpose.

Program orientation and guidance emerged as the main process variables in need of improvement. Topics for such training should include:

(1) Better preparation and ongoing support in areas related to intergenerational differences such as establishing reasonable expectations, setting boundaries, mediating different behavioral styles, and establishing the scope of the IGP from the outset.

(2) Time and stress management skills for YP to equip them with the tools to cope better with potentially new and stressful situations.

(3) Training in specific knowledge and skills required for meaningful participation in a particular IGP such as how to document and write a life story, help older adults with digital technology or be emotionally supportive of individuals who feel socially isolated and lonely, or who are victims of trauma such as the Holocaust.

Along with overarching issues, individual components that contribute to IGP success need to be considered. For example, YP's dissatisfaction with activity location was reflected in comments about the exhaustion they experienced due to commuting by public transport. Better geographical matching of participants and programs is another emerging need.

Future research is needed to develop guidelines for (a) comprehensive IGP training programs, including ongoing mentoring and specific training in areas relevant to various types of IGPs, (b) the reasonable allocation of time and resources for program duration, individual meeting duration, and to 

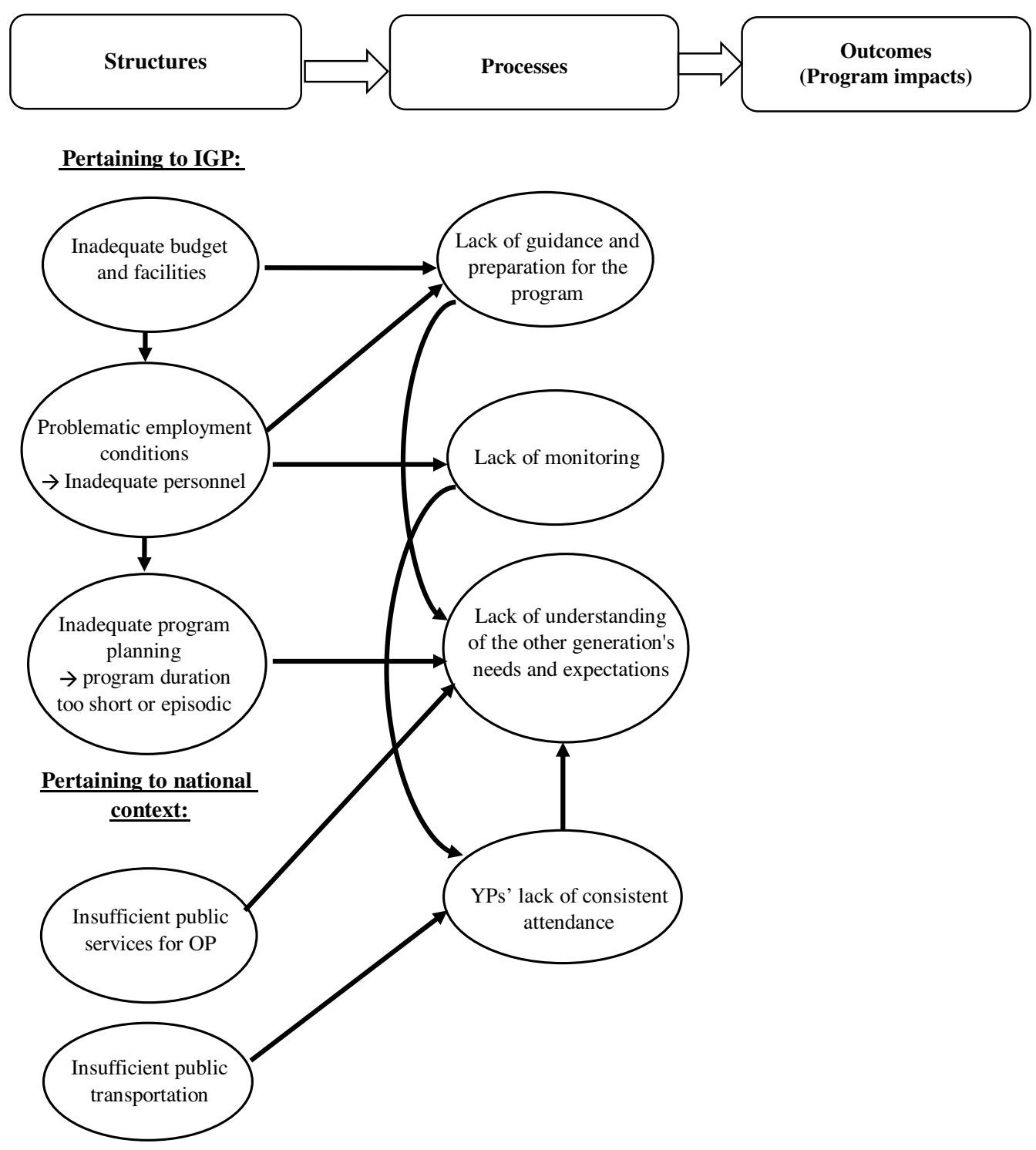

Figure 1. Structures and processes in need of improvement for intergenerational programs.

Notes: This paper focuses on processes and the structures that impact them. We examine study outcomes in another paper. While this figure describes examples of processes and structures, in the article, we also examine the differences in processes across different types of IGPs.

support organizers, and (c) to further investigate, monitor and improve programs - their processes and structures.

While we investigated processes, it became evident that many IGP shortcomings stem from systemic and institutional issues, as summarized in Figure 1. The lack of sufficient resources affects all areas from personnel, facilities, and equipment to the length of participation, the provision of training and mentoring, and monitoring of volunteers, all of which impact IGP quality and prospects for successful outcomes. This, in turn produces a vicious cycle, in which insufficiently supported programs produce lower satisfaction of participants, reduce program reputation, and therefore disincentivize appropriation of greater resources. This finding is in line with previous research that identified sustainable funding as a common need for improvement of IGPs (Ayala et al., 2007).

Some OP in the assistance group asked YP for help with basic personal and social services, which indicates an unmet need in this population. Such unmet needs seem to reflect a wider societal neglect of the older and frailer segments of the population that cannot be overcome by IGPs. The nature and extent of OP's unmet needs require a separate study. While IGPs have been shown to enrich older and younger participants' lives, they are but one aspect of a more comprehensive array of programs necessary to help older adults experience a better quality of life. 
Our research suggests that the concept of IGPs is sound, but that their processes and structures require strengthening.

One limitation of this study is the unequal distribution of participants across types of programs, such that assistance programs received more weight in the overall analysis (both in comparing OP to YP and in the qualitative analysis). Future studies should expand on this work by examining participants' preferences for change in processes underlying IGPs, and by examining IGP activities over time in real time, rather than through a retrospective approach. It is possible that the term "guidance" in our interviews was not sufficiently clear. For example, in arts programs, "guidance" may have been interpreted as guidance related to an art project rather than guidance on how to negotiate intergenerational relationships. Finally, since this study focused on how processes in IGPs can be improved, it tends to emphasize the negative aspects of IGPs, yet as stated above, we acknowledge that IGPs make valuable contributions to both older and younger adults' lives.

Our study provides two major contributions. First, in contrast with most IGP research which has involved program impact, this study focused on program processes and structures. While our analysis confirmed some previously reported process variables such as the need for training and guidance for participants (Lou and Dai, 2017), professional training for staff (Gigliotti et al., 2005), and sustainable funding (Ayala et al., 2007), it went on to describe the complex relationship between program types, processes, and structures (Figure 1). Even when program processes match goals, IGP activities may nevertheless fall short of their aspirations due to lack of funding, and factors external to the program, such as inadequate social services and insufficient public transit. These realities point to the need to take not only organizational structures into account, but also larger societal structures. Second, our study is the first to compare different types of IGPs based on content and goals. We found that different program types reflected different process variables. For example, since assistance programs focused on relationship building and one-on-one meetings, participants voiced a wish for longer periods of participation and more guidance emerged as a need in order to set realistic expectations, to anticipate and accept intergenerational differences, to learn how to respond to inappropriate requests, and how to improve interpersonal skills.

While all IGPs are committed to improving intergenerational relationships, each program takes place in a different context and must respond to different challenges. Consequently, programs need processes and structures unique to specific program types. Our research revealed that IGPs need to better meet the respective participant groups' needs, such as OPs' need for consistent scheduling, and YPs' need to balance their participation in IGPs with their personal responsibilities to family, education, or career. Our analysis of process variables and structures is a step toward a realistic view of what can be accomplished in IGPs within the current system and how to optimize new IGP initiatives.

\section{Acknowledgment}

We thank the Minerva Foundation and the Ministry of Senior Citizens in Israel for their support of this study. We thank the participants and organizers of IGP for contributing their time to this study and for sharing their perceptions of the programs.

\section{Conflict of interest}

The authors declare that there is no conflict of interest.

\section{Source of funding}

This work was supported by the Minerva Foundation and the Ministry of Senior Citizens in Israel.

\section{Description of authors' roles}

J. Cohen-Mansfield designed the study, supervised the data collection and analysis, and wrote the paper. A. Muff participated in the qualitative analysis and writing the article.

\section{Supplementary material}

To view supplementary material for this article, please visit https://doi.org/10.1017/S1041610221 000922.

\section{References}

Aguilera-Hermida, A. P., Anderson, E. A. and Negrón, V. A. (2020). Intergenerational activities that promote engaging conversations are preferred among young and older adults. Fournal of Intergenerational Relationships, 18, 71-87. doi: 10.1080/15350770.2019 .1608346 .

Ayala, J. S., Hewson, J. A., Bray, D., Jones, G. and Hartley, D. (2007). Intergenerational programs: perspectives of service providers in one Canadian city. 
fournal of Intergenerational Relationships, 5, 45-60. doi: 10.1300/J194v05n02_04.

Braun, V. and Clarke, V. (2006). Using thematic analysis in psychology. Qualitative Research in Psychology, 3, 77-101. doi: 10.1191/1478088706qp063oa.

Bullock, J. R. and Osborne, S. S. (1999). Seniors', volunteers' and families' perspectives of an intergenerational program in a rural community. Educational Gerontology, 25, 237-251. doi: 10.1080/036012799267855.

Canning, S. E., Gaetz, M. and Blakeborough, D. (2018). It takes time: building relationships and understanding through an intergenerational ballet programme. Dementia, 19, 270-284. 10.1177/1471301218772895.

Child, S. T. and Lawton, L. (2019). Loneliness and social isolation among young and late middle-age adults: associations with personal networks and social participation. Aging \& Mental Health, 23, 196-204. doi: 10.1080/13607863.2017.1399345.

Cohen-Mansfield, J., Hazan, H., Lerman, Y. and Shalom, V. (2016). Correlates and predictors of loneliness in older-adults: A review of quantitative results informed by qualitative insights. International Psychogeriatrics, 28, 557-576. doi: 10.1017/ s1041610215001532.

Cohen-Mansfield, J. and Jensen, B. (2017). Intergenerational programs in schools: prevalence and perceptions of impact. Fournal of Applied Gerontology, 36, 254-276. doi: 10.1177/0733464815570663.

Cohen-Mansfield, J. and Muff, A. (2021). Comparing community-based intergenerational activities: Participants, programs and outcomes. Manuscript submitted for publication.

Denzin, N. (1989). Interpretive Biography. Thousand Oaks, CA: Sage Publications.

Devore, S., Winchell, B. and Rowe, J. M. (2016). Intergenerational programming for young children and older adults: An overview of needs, approaches, and outcomes in the United States. Childhood Education, 92, 216-225. doi: 10.1080/00094056.2016.1180895.

Epstein, A. S. and Boisvert, C. (2006). Let's do something together. Fournal of Intergenerational Relationships, 4, 87-109. doi: 10.1300/J194v04n03_07.

Gaggioli, A., Morganti, L., Bonfiglio, S., Scaratti, C., Cipresso, P., Serino, S. and Riva, G. (2014). Intergenerational group reminiscence: A potentially effective intervention to enhance elderly psychosocial wellbeing and to improve children's perception of aging. Educational Gerontology, 40, 486-498. doi: 10.1080/ 03601277.2013.844042.

Gigliotti, C., Morris, M., Smock, S., Jarrott, S. E. and Graham, B. (2005). An intergenerational summer program involving persons with dementia and preschool children. Educational Gerontology, 31, 425-441. doi: 10.1080/03601270590928161.

Gualano, M. R., Voglino, G., Bert, F., Thomas, R., Camussi, E. and Siliquini, R. (2018). The impact of intergenerational programs on children and older adults: A review. International Psychogeriatrics, 30, 451-468. doi: $10.1017 / \mathrm{S} 104161021700182 \mathrm{X}$.

Holmes, C. L. (2009). An intergenerational program with benefits. Early Childhood Education fournal, 37, 113-119. doi: 10.1007/s10643-009-0329-9.
Jarrott, S. and Bruno, K. (2007). Shared site intergenerational programs: A case study. Fournal of Applied Gerontology, 26, 239-257. doi: 10.1177 10733464807300225.

Jarrott, S., Stremmel, A. J. and Naar, J. J. (2019). Practice that transforms intergenerational programs: A model of theory- and evidence informed principles. Fournal of Intergenerational Relationships, 17, 488-504. doi: 10.1080/ 15350770.2019.1579154.

Jeste, D. V., Lee, E. E. and Cacioppo, S. (2020). Battling the modern behavioral epidemic of loneliness: Suggestions for research and interventions. FAMA Psychiatry, 77, 553-554. doi: 10.1001/jamapsychiatry.2020.0027.

Kim, H. Y. (2017). Statistical notes for clinical researchers: Chisquared test and Fisher's exact test. Restorative Dentistry $\mathcal{E}$ Endodontics, 42, 152-155. doi: 10.5395/rde.2017.42.2.152.

Leedahl, S. N., Brasher, M. S., Estus, E., Breck, B. M., Dennis, C. B. and Clark, S. C. (2019). Implementing an interdisciplinary intergenerational program using the Cyber Seniors ${ }^{\circledR}$ reverse mentoring model within higher education. Gerontology E Geriatrics Education, 40, 71-89. doi: 10.1080/02701960.2018.1428574.

Lokon, E., Kinney, J. M. and Kunkel, S. (2012). Building bridges across age and cognitive barriers through art: College students' reflections on an intergenerational program with elders who have dementia. Fournal of Intergenerational Relationships, 10, 337-354. doi: 10.1080/ 15350770.2012.724318.

Lou, V. W. and Dai, A. A. (2017). A review of nonfamilial intergenerational programs on changing age stereotypes and well-being in East Asia. Fournal of Intergenerational Relationships, 15, 143-158. doi: 10.1080/15350770.2017. 1294427.

Maccallum, J., Palmer, D., Wright, P., CummingPotvin, W., Brooker, M. and Tero, C. (2010). Australian perspectives: Community building through intergenerational exchange programs. Fournal of Intergenerational Relationships, 8, 113-127. doi: 10.1080/ 15350771003741899.

Martins, T., Midão, L., Martínez Veiga, S., Dequech, L., Bisse, G., Bertram, M., McDonald, A., Gilliard, G., Orte, C., Vives, M. and Costa, E. (2019). Intergenerational programs review: Study design and characteristics of intervention, outcomes, and effectiveness. Fournal of Intergenerational Relationships, 17, 93-109. doi: 10.1080/15350770.2018.1500333.

Matthews, T., Danese, A., Wertz, J., Odgers, C. L., Ambler, A., Moffitt, T. E. and Arseneault, L. (2016). Social isolation, loneliness and depression in young adulthood: A behavioural genetic analysis. Social Psychiatry and Psychiatric Epidemiology, 51, 339-348. doi: 10.1007/ s00127-016-1178-7.

Mcalister, J., Briner, E. L. and Maggi, S. (2019). Intergenerational programs in early childhood education: An innovative approach that highlights inclusion and engagement with older adults. Fournal of Intergenerational Relationships, 17, 505-522. doi: 10.1080/15350770.2019. 1618777.

Meshel, D. S. and Mcglynn, R. P. (2004). Intergenerational contact, attitudes, and stereotypes of adolescents and older people. Educational Gerontology, 30, 457-479. doi: 10.1080/03601270490445078. 
Murayama, Y., Ohba, H., Yasunaga, M., Nonaka, K., Takeuchi, R., Nishi, M., Sakuma, N., Uchida, H., Shinkai, S. and Fujiwara, Y. (2015). The effect of intergenerational programs on the mental health of elderly adults. Aging $\mathcal{E}$ Mental Health Promotion Practice, 19, 306-314. doi: 10.1080/13607863.2014.933309.

Newman, S. and Hatton-Yeo, A. (2008). Intergenerational learning and the contributions of older people. Ageing Horizons, 8, 31-39.

Parkinson, D. and Turner, J. (2019). Alleviating social isolation through intergenerational programming: DOROT's summer teen internship program. Fournal of Intergenerational Relationships, 17, 388-395. doi: 10.1080/ 15350770.2019.1617606.

Perry, C. K. and Weatherby, K. (2011). Feasibility of an intergenerational Tai Chi program: A community-based participatory research project. Fournal of Intergenerational Relationships, 9, 69-84. doi: 10.1080/15350770.2011. 544215.

Pitman, A., Mann, F. and Johnson, S. (2018). Advancing our understanding of loneliness and mental health problems in young people. The Lancet Psychiatry, 5, 955-956. doi: 10.1016/S2215-0366(18)30436-X.

Richardson, J. T. (2011). Eta squared and partial eta squared as measures of effect size in educational research. Educational Research Review, 6, 135-147. doi: 10.1016/j .edurev.2010.12.001

Santini, Z. I., Jose, P. E., York Cornwell, E., Koyanagi, A., Nielsen, L., Hinrichsen, C., Meilstrup, C., Madsen, K. R. and Koushede, V. (2020). Social disconnectedness, perceived isolation, and symptoms of depression and anxiety among older Americans (NSHAP): A longitudinal mediation analysis. The Lancet Public Health, 5, e62-e70. doi: 10.1016/S2468-2667(19) 30230-0.
Steckler, A. and Linnan, L. (2002). Process evaluation for public health interventions and research. In: A. Steckler, \& L. Linnan (Eds.), Process Evaluation for Public Health Interventions and Research. San Francisco, CA: Jossey-Bass, pp. 1-23.

Stremmel, A. J., Travis, S. S., Kelly-Harrison, P. and Hensley, A. D. (1994). The perceived benefits and problems associated with intergenerational exchanges in day care settings. The Gerontologist, 34, 513-519. doi: $10.1093 /$ geront/34.4.513.

Sundström, A., Adolfsson, A. N., Nordin, M. and Adolfsson, R. (2020). Loneliness increases the risk of allcause dementia and Alzheimer's disease. The fournals of Gerontology. Series B, Psychological Sciences and Social Sciences, 75, 919-926. doi: 10.1093/geronb/gbz139.

Voglino, G., Gualano, M., Bert, F., Camussi, E., Thomas, R. and Siliquini, R. (2017). The impact of intergenerational programs on children and elder adults: Gianluca Voglino. European fournal of Public Health, 27. doi: 10.1093/eurpub/ckx186.023.

Ware, J. E., Jr. and Sherbourne, C. D. (1992). The MOS 36-item short-form health survey (SF-36). I. Conceptual framework and item selection. Medical Care, 30, 473-483.

Yasunaga, M., Murayama, Y., Takahashi, T., Ohba, H., Suzuki, H., Nonaka, K., Kuraoka, M., Sakurai, R., Nishi, M. and Sakuma, N. (2016). Multiple impacts of an intergenerational program in Japan: Evidence from the Research on productivity through intergenerational sympathy project. Geriatrics $\mathcal{E}$ Gerontology International, 16, 98-109. doi: 10.1111/ggi.12770.

Zucchero, R. A. (2010). Share your experience and I'll lend you my ear: Older adult outcomes of an intergenerational service-learning experience. Gerontology \& Geriatrics Education, 31, 383-402. doi: 10.1080/02701960.2010. 528275. 